\title{
Detection of spontaneous hemoperitoneum in a pregnancy complicated with endometriosis during caesarean section - a case report
}

\author{
Sonali Chauhan $^{1 *}$, Jiten Sharma ${ }^{1}$, Bhumika Arora ${ }^{1}$, Shivika Mittal ${ }^{2}$, Rita Mittal $^{1}$
}

\begin{abstract}
${ }^{1}$ Department of Obstetrics and Gynecology, Kamla Nehru State Hospital for Mother and Child, IGMC, Shimla, Himachal Pradesh, India

${ }^{2}$ Department of Obstetrics and Gynecology, Dr. Rajendra Prasad Government Medical College and Hospital, Tanda, Kangra, Himachal Pradesh, India
\end{abstract}

Received: 30 October 2020

Accepted: 09 December 2020

\section{*Correspondence: \\ Dr. Sonali Chauhan, \\ E-mail: sona.c.125@gmail.com}

Copyright: () the author(s), publisher and licensee Medip Academy. This is an open-access article distributed under the terms of the Creative Commons Attribution Non-Commercial License, which permits unrestricted non-commercial use, distribution, and reproduction in any medium, provided the original work is properly cited.

\begin{abstract}
Endometriosis is defined as the presence of endometrial glands and stroma outside the uterus. During pregnancy endometriosis and its related pain symptoms improve due to various metabolic, hormonal, immune and angiogenesis changes that occur in pregnancy. Spontaneous hemoperitoneum in pregnancy (SHiP) is a rare but serious pregnancy complication, which is associated with high rates of maternal and foetal morbidity and mortality. Endometriosis may be a potential risk factor for SHiP. Preformation of IVF on women with endometriosis may be a potential risk factor for SHiP. In gravid females with a history of endometriosis, severe abdominal pain, and a reduction of haemoglobin, physicians should always suspect SHiP because it is a life-threatening condition for both the mother and the baby. We are reporting a case of a primigravida with term gestation, taken up for emergency caesarean section in view of nonreassuring foetal heart rate. Intraoperatively there was evidence of hemoperitoneum and multiple endometriotic lesions on the posterior surface of uterus and Pouch of Douglas, Bilateral ovaries were adherent to the endometriotic growth on the posterior surface of uterus. Postoperatively, patient was stable and was discharged on postoperative day 4.
\end{abstract}

Keywords: Endometriosis, Pregnancy, Hemoperitoneum, Caesarean section

\section{INTRODUCTION}

Endometriosis is defined as the presence of endometrial glands and stroma outside the uterus. It affects $10 \%$ of women in the reproductive age group. ${ }^{1}$ During pregnancy endometriosis and its related pain symptoms improve because of arrest of ovulation, preventing bleeding of endometriotic tissue and also due to various metabolic, hormonal, immune and angiogenesis changes that occur in pregnancy. ${ }^{2}$ Spontaneous hemoperitoneum in pregnancy (SHiP) is a rare but serious pregnancy complication, which is associated with high rates of maternal and fetal morbidity and mortality. Importantly, the etiology of SHiP is still unclear. Notably, it is proposed that endometriosis may be a potential risk factor for SHiP. ${ }^{2}$ Here, we are reporting a case of spontaneous hemoperitoneum in a pregnancy with endometriosis, detected during caesarean section.

\section{CASE REPORT}

A 30-year-old primigravida, married for 10 months with spontaneous conception, without any history of infertility, was registered with us for ante natal care. At $35+6$ weeks of gestation she reported with complaints of pain abdomen. Her ultrasound was carried out and was found to be within normal limits. There were no signs and symptoms of patient being in labour and after reassurance to the patient she was advised to follow up one week later in outpatient department (OPD). At $36+6$ weeks of gestation she came with history of pain abdomen and was admitted. Her biophysical profile score was $8 / 10$ and a 
decision for induction of labour was taken. At 37 weeks of gestation, the patient was induced with dinoprostone gel intracervically. The cardiotocography tracing of the patient showed persistent foetal tachycardia. She was taken up for emergency lower segment caesarean section (LSCS) in view of non-reassuring foetal heart rate. On opening the abdomen, there was evidence of hemoperitoneum around $500 \mathrm{ml}$. Liquor was meconium stained. After the extraction of foetus, it was found that there was a jumbled-up mass on the posterior surface of uterus. Pouch of Douglas (POD) was completely obliterated by the mass. There was evidence of multiple endometriotic lesions in POD and posterior surface of uterus. Right fallopian tube and bilateral ovaries were adherent to the mass. There was e/o clots adherent to the surface of the mass but no active bleeding (Figure 1 and 2). Her course in the postoperative period was uneventful. Patient was discharged on postoperative day 4 .

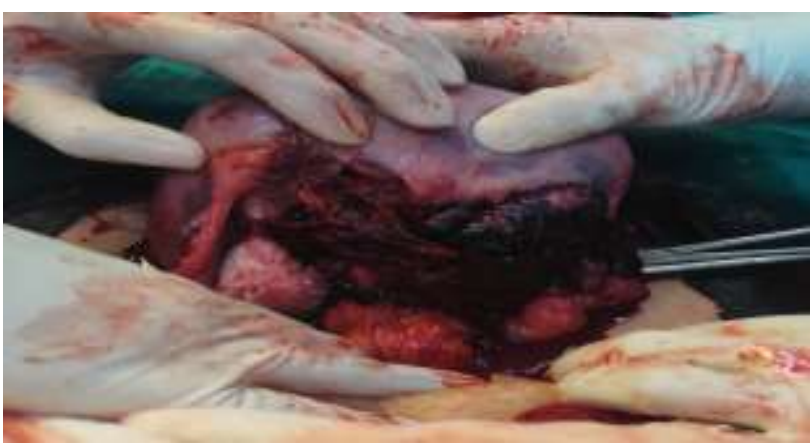

Figure 1: Type 4 endometriosis detected during LSCS with hemoperitoneum.

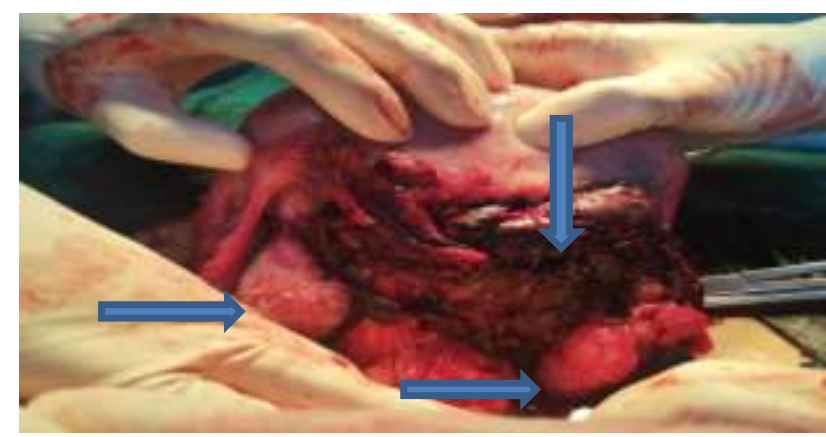

Figure 2: Pouch of Douglas completely obliterated with bilateral ovaries adherent to the mass. Clots are seen adherent to the mass.

\section{DISCUSSION}

Endometriosis is a benign disease defined by the presence of endometrial glands and stroma outside the uterus. The incidence of endometriosis in reproductive age group is $10 \% .120-25 \%$ of patients are asymptomatic. ${ }^{3}$ Risk factors associated with endometriosis are early menarche, short menstrual cycles, nulliparity, 1st degree relative, drug exposure to diethylstilbestrol (DES), dioxin and in younger age group it is usually associated with Mullerian anomalies and cervical or vaginal obstruction. 4 In the recent years the etiopathogenesis is also attributed to autoimmune disorder. ${ }^{4}$ Prolonged lactation and multiparity are protective. ${ }^{4}$

The clinical features of endometriosis are varied, and the presentation depends on the site of growth and severity of disease. Although usual presentation being six D's: dysmenorrhea (most common symptom), disorders of menstruation, dyspareunia, dyschezia, dysuria and dull aching chronic pain abdomen. Three types of endometriosis have been described: peritoneal superficial endometriosis, ovarian endometriomas, and deep infiltrating endometriosis (DIE). DIE usually involves the uterosacral ligaments, the rectovaginal space, and the upper third of the posterior vaginal wall, the bowel, and the urinary tract. ${ }^{4}$ Endometriosis is primarily found in the pelvis: on the ovaries most commonly, uterus, fallopian tubes, uterosacral ligaments, broad ligaments, round ligaments, cul-de-sac or ovarian fossa, as well as on the appendix, large bowel, ureters, bladder, or rectovaginal septum. Extra-pelvic locations of endometriosis are rare, but can include the upper abdomen, diaphragm, abdominal wall or abdominal scar tissue. ${ }^{4}$

SHiP is a rare but potentially life-threatening complication which occurs predominantly during the third trimester of pregnancy. ${ }^{5}$ SHiP is associated with adverse pregnancy outcomes for both mother and child, including stillbirth, neonatal mortality, and very low or low birth weight. ${ }^{2}$ The etiology of SHiP is still unknown but endometriosis seems to be a major risk factor. ${ }^{6}$ Controlled ovarian hyperstimulation plus embryo transfer may increase the severity or incidence of the rare condition known as SHiP. During the treatment of infertility, a high dosage of progesterone is used after in vitro fertilization and embryo transfer (IVF-ET), which can promote the process of decidualization. Thus, this treatment may result in extensive bleeding from the ectopic endometrium plant. Therefore, the influence of potential endometriosis should be considered during pregnancy, which may lead to vascular fragility. In addition, the use of forceps during the labor should also be cautious, as this treatment may further promote the rupture of endometriosis-induced defective serious vessels. Thus, we should pay more attention to the patients with forceps delivery, especially the patients with the history of endometriosis.

The suspicion that endometriosis is a possible risk factor for SHiP, was first suggested by Inoue et al in $1992 .{ }^{5}$ They explained the involvement of endometriosis in SHiP by two mechanisms: utero-ovarian vessels are more friable due to chronic inflammations associated with endometriosis, and resultant pelvic adhesions due to endometriosis in combination with enlargement of the uterus during pregnancy can place these vessels under greater tension, increasing the risk of rupture and bleeding. ${ }^{7}$

It has been shown that direct bleeding of endometriotic lesions may cause a hemoperitoneum. ${ }^{8}$ During pregnancy, 
endometriotic lesions become decidualised and therefore enlarge during the first trimester. Later in pregnancy these lesions can shrink, probably due to decidual necrosis and involution. $^{9}$ Because decidualised endometrium is dependent upon sustained progesterone signalling, failing progesterone levels or devascularisation can cause a weakening of the tissue. In endometriotic tissue, characterised by dysregulation of gene expression leading to progesterone resistance, 'functional' progesterone withdrawal can lead to necrosis of decidualised foci of endometriosis resulting in (gastrointestinal) perforation and bleeding of unpredictable severity. ${ }^{2}$

\section{CONCLUSION}

SHiP is a rare but potentially fatal complication for both pregnant women and their unborn babies. Preformation of IVF on women with endometriosis may be a potential risk factor for SHiP. A greater awareness of SHiP and its associated risk factors, such as pelvic endometriosis, may facilitate the diagnosis of this condition and expedite the intervention to improve maternal and fetal outcomes. We believe that in gravid females with a history of endometriosis, severe abdominal pain, and a reduction of hemoglobin, physicians should always suspect SHiP. Because SHiP is a life-threatening condition for both the mother and the baby, a prompt diagnosis must lead to prompt treatment. Hemoperitoneum was found during caesarean section in this patient with grade 4 endometriosis which adds this case to the small number of similar cases described.

Funding: No funding sources

Conflict of interest: None declared

Ethical approval: Not required

\section{REFERENCES}

1. Eskenazi B, Warner ML. Epidemiology of endometriosis. Obstet Gynecol Clin North Am. 1997;24(2):177-200.
2. Brosens IA, Fusi L, Brosens JJ. Endometriosis is a risk factor for spontaneous hemoperitoneum during pregnancy. Fertil Steril. 2009;92:1243-5.

3. Bulletti C, Ellisabetta M, Battistoni CS. Endometriosis and Infertility. J Assist Reprod Genet. 2010;27(8):441-7.

4. Tejaswini B, Chandushree, Kumar A, Bharati, Sumayya, Shruti K, et al. Incidental detection of atypical endometriosis during caesarean section. Endocrinal Metab Int J. 2018;6(5):324-86.

5. Inoue T, Moriwaki T, Niki I. Endometriosis and spontaneous rupture of utero-ovarian vessels during pregnancy. Lancet. 1992;340:240-1.

6. Ginsburg KA, Valdes C, Schnider G. Spontaneous utero-ovarian vessel rupture during pregnancy: three case reports and a review of the literature. Obstet Gynecol. 1987;69:474-6.

7. Passos F, Calhaz-Jorge C, Graça LM. Endometriosis is a possible risk factor for spontaneous hemoperitoneum in the third trimester of pregnancy. Fertil Steril. 2008;89:251-2.

8. Katorza E, Soriano D, Stockheim D, Mashiach R, Zolti M, Seidman DS, et al. Severe intraabdominal bleeding caused by endometriotic lesions during the third trimester of pregnancy. Am J Obstet Gynecol. 2007; 197:501.

9. McArthur JW, Ulfelder H. The effect of pregnancy upon endometriosis. Obstet Gynecol Surv. 1965;20:709-33.

Cite this article as: Chauhan S, Sharma J, Arora B, Mittal S, Mittal R. Detection of spontaneous hemoperitoneum in a pregnancy complicated with endometriosis during caesarean section - a case report. Int J Reprod Contracept Obstet Gynecol 2021;10:401-3. 